\title{
MATERNIDAD, FEMINIDAD, SEXUALIDAD. ALGUNOS ASPECTOS DE LAS PRIMERAS JORNADAS EUGÉNICAS ESPAÑOLAS (MADRID, 1928-MADRID, 1933)
}

por

MARIE-ALINE BARRACHINA

Université de Nice-Sophia Antipolis

RESUMEN: Esta contribución estudia las actas de las Primeras jornadas eugénicas españolas, que tuvieron lugar en la primavera de 1933, después de un primer intento abortado en 1928. El estudio de los dos volímenes de estas actas evidencia el entramado ideológico y político, en los años veinte y treinta, del debate científico —-médico y jurídico- sobre la sexualidad. En 1928, la dictadura de Primo de Rivera interrumpe por «regodeo pornográfico» la serie de conferencias previstas, cortando así de raíz el debate socio-político sobre un problema urgente de sociedad: la planificación de la natalidad. El segundo congreso, en los últimos meses del bienio reformador, revela las distancias abismales que separan hasta los aparentemente más próximos politica e ideológicamente. Desgajado el sexo de las esferas privadas del confesional y de la alcoba, se plantea la cuestión de los límites éticos y jurídicos de la intervención del Estado, y no dejan de apuntar tesis de indudable tinte totalitario, al lado de otras bumanistas y feministas, modernísimas todas. Diferencia sexual e identidad, maternidad y sexualidad, emancipación femenina y emancipación sexual son temas que, al remover la posibilidad de otro orden sexual, bacen vislumbrar a los congresistas los contornos de otro orden social inédito y angustioso, hasta para los más progresistas.

Palabras Clave: Eugenesia. Feminismo. Birth control. Medicina. Derecho. Dictadura. Segunda República.

ABSTRACT: This article studies the proceedings of the "Primeras jornadas eugenicas españolas», which took place in the spring of 1933 after an abortive first attempt in 1928. Study of the two volumes of proceedings provides evidence of the ideological and political framework of the medical and juridical debate about sexuality in the 1920s and 1930s. In 1928, the Primo de Rivera dictatorship intervened to prevent the «pornographic indulgence" represented by the series of planned conferences, cutting off at the root social and political debate on an urgent problem in Spanish society: birth control and family planning. The second congress, in the final months of the «bieno refor- 
mador» (1931-1933), reveals the abyss that separated even those groups who appeared politically and ideologically closest. Now that sexuality bad been removed from the confession booth, the question of the etbical and juridical limits of state intervention could be raised, and there was little besitation in expressing viewpoints with an unmistakably totalitarian colour, alongside humanist and feminist perspectives, all of them extremely modern. Sexual difference and identity, maternity and sexuality, and female and sexual emancipation, were themes that, by raising the possibility of another sexual order, allowed the participants in the congress to glimpse the contours of a world that was unprecedented and shocking, even for the most progressive.

KEY WORDS: Dictatorship. Second Republic. Feminism. Birth Control. Family planning. Medical discourse. Juridical discourse.

Para esta contribución, se estudian básicamente los dos volúmenes de las actas de las Primeras jornadas eugénicas españolas. Esta obra fue publicada en Madrid en 1934 por el editor Javier Morata con el título de Genética, Eugenesia y Pedagogía sexual ${ }^{1}$. Estos dos volúmenes ya han sido el objeto de varios trabajos ${ }^{2}$, y hace ya bastante tiempo tuve también la ocasión de redactar un primer esbozo de estudio ${ }^{3}$. Quienes por casualidad han podido hojear aquella publicación me perdonarán algunas repeticiones imprescindibles para la claridad del propósito, ya que si se aborda el estudio con una perspectiva bastante distinta, no dejan de ser idénticos los cimientos de la reflexión.

En un trabajo general sobre la historia de la sexualidad en la España contemporánea, el estudio de los dos acontecimientos que narran estas actas de congreso se adentra en un debate que apenas si acababa de salir entonces del secreto de los consultorios médicos, de las alcobas y de los confesionales.

Por cierto, no era nuevo en España el interés por un análisis científico, social y político de la sexualidad y de sus incidencias en la salud pública. Sólo basta con recordar algunos títulos como los siguientes: El amor en España, características masculinas, publicado en Madrid en 1927 por César Juarros; Libertad de amar y derecho a morir (1928), colección de conferencias pronunciadas en la Universidad de Montevideo en 1925 por Luis Jiménez de Asúa; Teología y

1 Noguera, Enrique y Huerta, Luis (Eds.): Genética, Eugenesia y Pedagogía sexual. Libro de las primeras jornadas eugénicas españolas, Madrid, Javier Morata, Editor, 1934, 2 t., 450 p. y 412 p.

2 Pérez SANZ; Pilar y BRU Ripoll, Carmen: «La Sexología en la España de los años 30. Tomo I: las Jornadas eugenésicas de 1928 y 1930», Revista de Sexología (Madrid), nº 30, 1987, 89 p.; ÁLVAREZ PELÁEZ, Raquel: "Origen y desarrollo de la eugenesia en España», en SÁNCHEZ RON, José Manuel (Ed.): Ciencia y Sociedad en España, Madrid, El Arquero y C.S.I.C., 1988, pp. 179-204; MOReNo Mengíbar, Andrés y VÁzQuez García, Francisco: "Crisis nacional, eugenesia y regeneracionismo biológico en España, 1898-1936», Gades (Cádiz), nº 23 (Actas del Congreso "1898-1998 Balance de su siglo» Abril, 1998), 1999, pp. 203-213.

3 BARRACHINA, Marie-Aline: «Las primeras jornadas eugénicas españolas (Madrid 1928-Madrid 1933)", en Hommage à Nelly Clemessy, Nice, Université de Nice-Sophia Antipolis (Publications de la Faculté des lettres, Arts et Sciences Humaines de Nice, nueva serie, $n^{\circ} 14$ ), 1993, pp. 43-55.

Hispania, LXIV/3, núm. 218 (2004) 1003-1026 
Eugenesia, de Jaime Torrubiano Ripoll (Madrid, 1929); El problema higiénico, punto de vista de una mujer moderna, por Hildegart (Carmen Rodríguez Carballeira), Madrid, 1930; Los niños mentalmente anormales, de Gonzalo Lafora (1930); La sexología, ensayos, de Quintiliano Saldaña (Madrid, 1930). Sin olvidar, en los primerísimos años veinte, las numerosas producciones de Gregorio Marañón, y en particular los numerosos artículos publicados en El Siglo Médico (por ejemplo «Biología y Feminismo», conferencia pronunciada en Sevilla en 1920), o en la Revista de Occidente.

Sin embargo, aquellas jornadas de debate y de divulgación científica se distinguen de las publicaciones e intervenciones anteriormente evocadas en la medida en que pretenden abrirse a la mayoría, y que se trata ahí, explícitamente, de romper el silencio y el doble lenguaje tradicional imperante en cuanto se trata de sexualidad y de procreación. Parece pues necesaria una mínima contextualización de aquellos dos acontecimientos y de la publicación subsiguiente.

Se evocarán pues, primero las circunstancias de las primeras jornadas y luego de las segundas indicando, en su caso, la identidad de los protagonistas. Se indicarán luego algunos datos sobre la composición de los dos volúmenes, ya que dicha composición es significativa de la postura de los organizadores. De este modo, se esbozará un panorama de los temas abordados, y se extraerán las problemáticas básicas.

\section{LAS DIFICULTADES DEL PRIMER CONGRESO}

Un artículo que aparece como complemento documental al final del volumen $\mathrm{II}^{\circ}$ de Genética, eugenesia y pedagogía sexual proporciona valiosas informaciones acerca de las circunstancias y avatares del primer congreso ${ }^{4}$. Enrique Noguera ya había publicado este artículo titulado «Cómo se yuguló la generosa idea del Primer Curso Eugénico Español» en 1930, en el n 50 de la Gaceta Médica Española de la que era entonces el director. Por el testimonio directo de Noguera, nos enteramos de que las primeras «Jornadas» habían sido organizadas en 1928 por iniciativa de la Gaceta Médica Española, en el recinto de la Facultad de Medicina de San Carlos, con el asentimiento de varias entidades de prestigio en los ámbitos científicos, como el Colegio de Doctores de Madrid, la Sociedad Española de Biología, la Sociedad Española de Antropología y la Sociedad Ginecológica Española.

El programa fue inaugurado en la sede de la Sociedad de los Amigos del Niño, por Julio Noguera, "profesor de Anormales del Estado y de la Benefi-

4 Noguera, Enrique: «Datos para la historia contemporánea (1). Cómo se yuguló la generosa idea del Primer Curso Eugénico Español», en Genética, Eugenesia y Pedagogía sexual, op. cit., t. II, pp. 399-412. Ver NASH, Mary: "Aproximación al movimiento eugénico español: el primer curso eugénico español y la aportación del Dr. Sebastián Recaséns", Gimbernat. Revista catalana d'Història de la Medicina $i$ de la Ciència (Barcelona), vol. IV (1985), pp. 195-202.

Hispania, LXIV/3, núm. 218 (2004) 1.003-1026 
cencia municipal de Madrid», que anunció que la temática general de las sesiones se organizaba en torno a «La defensa de la raza en el niño». El ciclo de las ponencias empezó el 2 de febrero de 1928 y debía clausurarse el 12 de abril, después de pronunciarse unas diez conferencias. El programa era el siguiente:

- 2 de febrero de 1928: Eugenesia y procreación, por Sebastián Recaséns, ginecólogo, catedrático de obstétrica y ginecología y decano de la $\mathrm{Fa}$ cultad de Medicina de Madrid. Recaséns fue además presidente de la Academia Nacional de Medicina y director, en 1932, de la Maternidad Santa Cristina;

- 9 de febrero de 1928: El aspecto jurídico de la maternidad consciente, por Luis Jiménez de Asúa. catedrático de derecho penal. Jiménez de Asúa es conocido, sobre todo, por sus opiniones socialistas y por sus actividades como político. Entre otras actividades, consta que fue presidente de la Comisión parlamentaria encargada de la elaboración del texto constitucional de 1931 (del 29 de julio al 29 de agosto de 1931);

- 16 de febrero de 1928: Los niños que vemos en nuestros hospitales. Medidas eugenésicas que reclaman los pediatras, por José Estella, catedrático de pediatría;

- 23 de febrero de 1928: Pensamiento católico ante los problemas eugenésicos, por el R. P. José A. de Laburu, de la Compañía de Jesús. Nada más empezar la campaña de hostilidad a las Jornadas, el padre Laburu renunció a pronunciar su conferencia;

- $1^{\circ}$ de marzo de 1928: La maternidad y el infanticidio ante el derecho por Joaquín Noguera, abogado, catedrático de filosofía y letras;

- 8 de marzo de 1928: Neuropatías producidas o influenciadas por la procreación excesiva o patológica en la pobreza del medio, por José Sanchís Banús. Neurólogo en el Hospital provincial de Madrid, era militante del Partido Comunista de España, y fue diputado en 1931;

- 15 de marzo de 1928: Bases y pruebas demográficas de la Eugenesia por Luis de Hoyos, catedrático de fisiología (pero no aparece ninguna huella de esta ponencia en las actas);

- 22 de marzo de 1928: Non moechaberis; discreteo ético-psíquico-religioso sobre la urgencia de la sensualidad, por el R. P. Francisco Sureda (Vicariato Castrense). La ponencia fue suprimida por orden de la jerarquía eclesiástica poco antes de la prohibición oficial de las Jornadas;

- 29 de marzo de 1928: El problema de la maternidad en España, por Gregorio Marañón, médico del Hospital provincial de Madrid (conferencia suprimida);

- 12 de abril de 1928: Aspectos sociales de la procreación: medidas eugenésicas de buen gobierno aplicables en España, por Ángel Ossorio y Gallardo, abogado, militante conservador (conferencia suprimida). 
Como se puede comprobar, los organizadores proponían un programa equilibrado, con prestigiosos especialistas del Derecho y de la Medicina con sus especialidades más directamente vinculadas con el tema. También habían buscado el aval de la autoridad religiosa, invitando a dos representantes de la jerarquía intelectual de la Iglesia. Por fin, conviene advertir que la mitad de los invitados, por lo menos, profesaban explícitas convicciones políticas o religiosas que, más allá de sus competencias científicas, les conferían un deber moral de reflexión sobre las implicaciones sociales, políticas, económicas y éticas de sus conclusiones técnicas.

Las dos primeras ponencias, al parecer, no sufrieron trabas, y la tercera, la del pediatra José Estella, cuenta con una reseña bastante halagüeña en el diario El Debate del 17 de febrero de 1928. Sin embargo, la conferencia pronunciada la semana anterior por el Profesor Luis Jiménez de Asúa sobre «El aspecto jurídico de la maternidad consciente» no dejó indiferente al diario católico conservador. La contraofensiva empieza pues con un ligero retraso, el $18 \mathrm{de}$ febrero, en la sección del diario titulada «Lo del día». A partir de esta fecha, el "curso eugénico", que hasta entonces había contado con poca publicidad por parte de El Debate (reseñas en la sección La vida en Madrid, p. 5) ya no desaparèce de la primera página.

Con el título explícito «De lo científico a lo erótico» (18 de febrero), el editorialista denuncia las palabras pronunciadas en la Facultad de Medicina, y acusa a los organizadores de las jornadas. En el artículo que ya evocamos más arriba, Enrique Noguera señala que la campaña de hostilidad se dedicó principalmente a provocar desacuerdos entre los organizadores. Una prueba de ello aparece en este primer artículo de El Debate del 18 de febrero. En efecto, a la polémica general se añade otra más específica entre el diario de la derecha católica conservadora y el doctor Zúñiga Cerrudo, secretario del Colegio de Doctores que firmó el apoyo a las jornadas. El 20 de febrero, el doctor Zúñiga quiso desresponsabilizar a su Colegio en cuanto a la organización de las conferencias. El Debate contesta que aprobar viene a ser lo mismo que organizar, y exige del Colegio de Doctores una rotunda condena de las jornadas (intercambio de los 18 y 20 de febrero).

Mientras tanto, los organizadores, que contaban con él para dar a las jornadas un aval eclesiástico, reciben del padre Laburu una carta de renuncia. El padre Laburu tenía que pronunciar su ponencia el 23 de febrero. Aquel mismo 23 de febrero, El Debate publicaba en la sección "Lo del día» un artículo titulado "Contra la pornografía» que evocaba una nota del gobierno en la que se recomendaba el estricto respeto de la Real orden del 12 de noviembre de 1927 sobre pornografía. Sin preocuparse demasiado de las contradicciones -0 al revés muy satisfecho-, El Debate anunciaba también aquel mismo 23 de febrero, con palabras muy halagüeñas, un homenaje al Profesor Marañón previsto para el 25 de febrero, homenaje en el que debían participar, amén del propio Marañón, los profesores Recaséns y Sanchís Banús. 
La campaña organizada por El Debate y El Siglo Futuro encuentra su segundo impulso en los primeros días del mes de marzo, con la conferencia de Joaquín Noguera a quien se tacha de poco riguroso científicamentes. El furor de la prensa católica llega a su colmo cuando Luis Jiménez de Asúa, que el 9 de febrero había dado en San Carlos su conferencia sobre «La maternidad consciente», reanuda la pelea un mes escaso después en la Universidad de Murcia, con una conferencia sobre «Los aspectos jurídicos de la selección humana». Ya es demasiado para la derecha católica que basa su campaña en la recatada y virtuosa condena de una supuesta propaganda erótica, o incluso pornográfica. El 14 de marzo, El Debate se escandaliza, denuncia la subversión de la moral y del orden social, estigmatiza el amor libre y sus «aberraciones» y concluye:

«No es discutible [...] la posición del conferenciante. Decididamente es un predicador del amor libre con todas sus aberraciones disolventes. Pero hay aún más. Sobre la doctrina y el fárrago científico propaga la frase erótica: [...] Por decoro no publicamos las frases pronunciadas en la Universidad de Murcia [... $\rrbracket^{* 6}$.

Para la derecha católica - y por cierto, esto no es nada sorprendentecualquier ponencia pública acerca del cuerpo, cualquier consideración acerca del proceso fisiológico que aboca a la maternidad participa de la culpa y del pecado. Sin embargo, más que la cuestión científica y humana, lo que preocupa a los oponentes a las jornadas eugénicas de 1928 es su carácter subversivo del orden familiar y social. El Debate revela las razones políticas profundas de la campaña en un corto artículo del 16 de marzo que subraya la convergencia de puntos de vista entre las tesis defendidas por Jiménez de Asúa y las que defiende el periódico El Socialista ${ }^{7}$.

Huelga decir que el debate es más político que ético, a pesar de las denegaciones de El Debate. Es de notar, además, que las palabras de los juristas son las que más escandalizan a los periodistas de El Debate, cuando al revés las cuestiones éticas con regusto un tanto racista que debería suscitar la conferencia de Recaséns - ya la evocaremos más adelante- no inspiran la más mínima emoción entre los redactores del diario católico.

Finalmente, sólo pudo ser pronunciada media docena de conferencias. En los días que precedieron la conferencia de Gregorio Marañón, programada para el 23 de marzo, una nota firmada por el Ministro de Instrucción Pública Callejo prohibió el Curso Eugénico, acusando a los organizadores de fomentar «el regodeo pornográfico». El Debate saludó aquella medida con un entusiasmo pretendidamente razonable. A continuación, el profesor Jiménez de Asúa es dado de baja, lo que provoca una fuerte protesta estudiantil.

5 «Lo del día: «iEscandaloso!», El Debate (Madrid), 2 de marzo de 1928.

6 «Lo del Día: «Predicadores del amor libre», El Debate (Madrid), 14 de marzo de 28.

7 «Lo del Día: «Una coincidencia», El Debate (Madrid), 16 de marzo de 1928. 
Así fue cómo la hostilidad de la derecha católica acabó con aquel primer intento en España de reflexión universitaria, institucional, colectiva y pluridisciplinaria sobre las cuestiones eugénicas. Con el pretexto de impedir estériles devaneos pornográficos, la derecha católica consiguió estorbar e incluso impedir el desarrollo de una reflexión fecunda sobre un tema tan eminentemente social, político y económico como el de la planificación familiar y de la salud pública.

\section{8: LA PLANIFICACIÓN FAMILIAR EN EL CENTRO DEL DEBATE}

En nombre de la lucha contra la mortalidad infantil, los juristas Jiménez de Asúa y Joaquín Noguera se proclamaban partidarios de lo que entonces se llamaba la «maternidad consciente». Como el médico Gregorio Marañón que imponía entonces sus autoridad en cuestiones de sexualidad femenina, Jiménez de Asúa y Noguera defienden que una nueva ética basada en la prioridad del derecho de los individuos ya es posible gracias al progreso técnico, y que esta nueva ética debe sustituirse a la ética tradicional basada en la cantidad, la cual no se justifica más que por el interés de una minoría. Esta tesis es la que defenderán algunos en 1933, en particular Matilde de la Torre en su ponencia sobre «Feminismo y Pacifismo», e Hildegart en su larga conferencia sobre «Neomaltusianismo y Maternidad consciente».

Como modelo prioritario del eugenismo, Asúa y Noguera proponen el derecho a la limitación de los nacimientos, limitación que permitiría garantizar la protección de la salud de las mujeres y de los niños. Los dos reclaman también medidas de protección contra el contagio venéreo. Así Jiménez de Asúa, que propone la penalización del contagio por vía sexual. Con este mismo propósito de protección de la madre y del niño, Joaquín Noguera reclama la creación de un certificado prenupcial. Los dos, por fin, son partidarios del aborto terapéutico, pero ninguno de los dos se adentra en la vía de la selección de tipo racial. Para resumir, los dos son partidarios de un eugenismo suave, tan respetuoso de los intereses de los individuos como de los intereses de la especie.

Adoptando un punto de vista totalmente opuesto, el doctor Sebastián Recaséns anuncia de inmediato su adhesión a unas tesis claramente racistas:

«Está comprobado que los cruces entre razas superiores dan tipos superiores y viceversa. Así ha logrado mejorar el tipo racial América del Norte, adonde emigraron, no los peores, sino los más selectos, quizá, de varios países en tiempos de persecuciones políticas y religiosas. En Australia y América del Sur sucedió algo semejante, si bien los latinos, por haber sido menos puros que los sajones, no han logrado alcanzar el nivel eugénico que enorgullece a los primeros. En Centroamérica los españoles procrearon sin ningún criterio selectivo, dando origen a un tipo mulato inferior, que en las luchas contra la metrópoli fueron nuestros mejores enemigos»8.

8 RECASÉNS, Sebastián: «Eugenesia y procreación», en Genética, Eugenesia y Pedagogía sexual, op. cit., t. II, pp. 328-329.

Hispania, LXIV/3, núm. 218 (2004) 1003-1026 
Teniendo en cuenta estas convicciones, se comprenderá que para Recaséns, cuando no se debe a la miseria, la mortalidad infantil sólo se debe a uniones sexuales ineptas. Cree en las virtudes de lo que él llama «la unión eugénica», y considera que la limitación de los nacimientos no se justifica si existe una verdadera política de protección por parte del Estado. Insensible a los riegos de agotamiento fisiológico, ya que afirma sin inmutarse que «contando corto, la mujer puede tener 15 o 20 hijos», Recaséns no rechaza la limitación de los nacimientos para las madres potenciales que sufren alguna enfermedad:

«Se debe impedir que sean muchas veces madres aquellas que por enfermedades cardíacas, renales, tuberculosas, sífilis terciaria, neurosis graves, pueda representar cada hijo que viene un factor destructivo de la madre y, por tanto, del elemento más indispensable para el buen desarrollo del recién nacido y en algunas ocasiones, como en las últimas enfermedades citadas, motivo de positivos trastornos generativos y venida al mundo de seres enfermos o depauperados»?

Invocando el aval científico de Mendel, el doctor Recaséns confiere a las prácticas matrimoniales más tradicionales los colores de la modernidad ya que traslada al terreno del eugenismo las políticas tradicionales de las alianzas entre familias de ambientes y fortunas similares. De esta manera, propone confirmar la legitimidad eugénica de tales alianzas matrimoniales basadas en la elección de cónyuges de herencia conocida:

«Mendel explicó el mecanismo por el cual la herencia hace los hijos copartícipes de las cualidades paternas [...]. Es evidente que pueden venir de razas puras elementos mejores, y cabe una selección que vaya paulatinamente redimiendo de taras hereditarias a la humanidad. [...] El hecho patente es que de padres-bien salen hijos-bien» 10 .

¿Qué recomendación hay más tranquilizadora para un público conservador que ésta que preserva el orden familiar y social a la par que legitima la segregación social y nacional? De ahí que el discurso de Recaséns pase relativamente desapercibido en 1928, en la medida en que sólo propone la sustitución de una instancia represiva religiosa por una instancia represiva médico-jurídica. Se encontrará de nuevo esta tesis en el congreso de 1933, especialmente en la conferencia del jurista Quintiliano Saldaña.

9 Ibid., p. 331.

10 lbid., p. 329.

Hispania, LXIV/3, núm. 218 (2004) 1003-1026 


\section{3, EL SEGUNDO CONGRESO Y LA PUBLICACIÓN DE LAS JORNADAS}

En cuanto cayó la dictadura de Primo de Rivera, la sección San Carlos de la F.U.E., luego la prensa liberal, así como socios del Ateneo de Madrid y universitarios (Fernando de los Ríos, José Ortega y Gasset, etc.) apoyaron el proyecto de un nuevo congreso sobre eugenismo. Sin embargo, hubo que esperar el final de la primera legislatura de la Segunda República para que aquel proyecto viera la luz, con el apoyo del Ministro Fernando de los Ríos.

Las primeras Jornadas eugénicas españolas se verificaron por fin en la primavera de 1933 (finales de abril - primeros de mayo). Bajo la presidencia del jurista Fernando de los Ríos, entonces todavía Ministro de Instrucción Pública, reúnen a unos cincuenta participantes. El programa de las Jornadas, transcrito en las primeras páginas de las actas ${ }^{11}$, anuncia unos setenta y cinco estudios, distribuidos en dos grandes apartados: doce ponencias a cargo de prestigiosas personalidades políticas, científicas, literarias y periodísticas tenían que proponer unos puntos de vista generales sobre diferentes aspectos de la cuestión eugénica: sexualidad, amor, natalidad, feminismo, pacifismo, moral cristiana y nueva moral, etc., y veinticuatro cursos más técnicos debían desarrollarse a continuación.

Dichos cursos podrían clasificarse en tres grupos. Había primero cursos generales sobre genética (cursos 1 y 2), antropología y ecología (cursos 3 y 4), selección biológica y birth control (cursos 5,6 y 7); abolicionismo y prostitución (curso 8), y patología del trabajo y de la miseria (curso 9).

Seguían cursos más especializados sobre puericultura y obstétrica (cursos 10 y 11), patologías y eugenismo (cursos 12 a 18: sífilis, tisis, cáncer, endocrinología, hematología, neurología, psicoanálisis).

Los últimos cursos consideraban la eugenesia en sus relaciones con otras disciplinas: higiene, pedagogía, derecho, historia, literatura, y por fin política (cursos 19 a 24).

El libro de las actas no respeta del todo el orden anunciado en el programa. Presenta cuatro secciones y un prólogo que contiene las tres ponencias inaugurales, seguidas por ocho ponencias (y no doce, como lo anunciaba el programa) reunidas bajo el título común de «Curso social». Vienen luego treinta y cuatro contribuciones «técnicas»y, para concluir, un apéndice con las cinco conferencias de 1928 y el resumen histórico redactado por Enrique Noguera.

Se apuntarán, sin sacar conclusiones, algunos notables ausentes, como Gregorio Marañón que debía intervenir sobre «Sicopatología del amor» y Sebastián Recaséns que debía hablar acerca de «Regulación de la natalidad». Sus conferencias, como la de Fernando Valera sobre «Hacia una moral sincera», figuran en el programa inicial.

\footnotetext{
11 «Programa de las Primeras Jornadas eugénicas españolas Madrid, 1933», en Genética, Eugenesia y Pedagogía sexual, op. cit., t. I, pp. XIX-XXIV.
} 
Para terminar con este apartado muy descriptivo, tenemos que apuntar algunas indicaciones sobre los participantes. Entre ellos hay, lógicamente, una mayoría de miembros de las profesiones médicas y paramédicas (seis fisiólogos, dos ginecólogos, siete higienistas, un genético, un biólogo), a los cuales cabe añadir a tres veterinarios y a un biólogo vegetal. Se trata en total de unos treinta médicos profesionales. Los demás participantes son juristas, publicistas y escritores de mayor o menor prestigio y notoriedad.

\section{PRoblemática DEL SEGUNDO CONGRESO: NUEVO ORDEN SEXUAL Y NUEVO ORDEN SOCIAL}

En su ponencia inaugural titulada «Hacia una nueva cultura del Amor», el profesor Luis Huerta recuerda que de los cuatro campos básicos del eugenismo (biológico, médico, social, pedagógico), el tercero, el social, es seguramente el más preocupante:

«Es esta zona del conocimiento donde más se encrespan las pasiones para producir la asfixia de los ideales eugénicos, y es aquí, por tanto, donde había que proceder con más escrupulosa pulcritud, llamando a informar a las personalidades más destacadas del Derecho y de la Política, de la Historia y de la Literatura, organizándose para ello los cursos de regulación de la natalidad (birth control), prostitución y abolicionismo, patología del trabajo y de la miseria, derecho, política, historia y literatura. Con esta vasta perspectiva las Jornadas eugénicas realizan por primera vez en España una labor única en su género»12.

Al decir esto, Luis Huerta establecía implícitamente una jerarquía de las prioridades, y señalaba las cuestiones más importantes a los ojos de los organizadores: control de la natalidad, problema de la prostitución, patologías relacionadas con el trabajo y la miseria. Dicho de otro modo, problemas eminentemente sociales. Aparece pues claramente que lo esencial del debate se situaba en el terreno de la salud pública y del derecho. Como en 1928, la ideología estaba en el centro del discurso científico. Pero ya no se trataba solamente de militar a favor del birth control con el propósito de preservar la salud de las madres y de los niños o de imponer una ética médica que se inmiscuiría en la intimidad familiar o individual. También se trataba de debatir del grado de oportunidad del eugenismo y de la pertinencia de sus bases. Se trataba de definir el grado de competencia de las entidades colectivas (la medicina, el derecho, las autoridades religiosas, etc.) en términos de control, no sólo de cantidades, sino también de calidad de los niños por nacer. Se trataba pues de pensar seriamente el papel de la pulsión sexual y del amor en el proceso procreador.

12 Huerta, Luis: «Hacia una nueva cultura del Amor», Ibid., t. I, p. 10. 
Si la cuestión de la sexualidad y de la identidad femenina no es «el tema» fundamental de aquellas jornadas sobre el eugenismo, no cabe duda sin embargo que dicha cuestión se encuentra ineludiblemente relacionada con el tema general del congreso. De los prejuicios y de las ideas que van desarrollando los participantes sobre la sexualidad femenina derivan casi necesariamente sus tesis sobre el eugenismo, la contracepción o el papel social de las mujeres en relación con su papel de madres efectivas o potenciales. Naturalmente, todas las conferencias no abordan con la misma intensidad esta cuestión, y por ello me pareció oportuno seleccionar, entre el medio centenar de contribuciones, las que me parecieron más representativas.

Con esta perspectiva, dejé de lado las contribuciones muy precisas (unas quince contribuciones) sobre patologías particulares - aun cuando esas contribuciones tomaban en cuenta consideraciones sociales o económicas. También dejé de lado las contribuciones sobre el mundo animal y vegetal (son tres). Aparté por fin unas quince contribuciones muy generales que, por varias razones, no abordan la cuestión de los roles sexuales. Quedan siete de las ocho ponencias sobre el «ciclo social» y media docena de contribuciones «técnicas». Entre los autores de los textos que constituyen este corpus, nos encontramos con cinco médicos, cinco escritores y tres juristas. El lector encontrará en las líneas que siguen la lista de dichos participantes.

Entre los juristas, nos encontramos con Jaime Torrubiano Ripoll, catedrático de Derecho canónico, diputado en las Cortes Constituyentes de 1931, quien pronuncia la tercera ponencia sobre «El Cristianismo es el mejor auxiliar de la Ciencia eugénica» ${ }^{13}$. También jurista, Mariano Ruiz Funes, catedrático de Derecho en la Universidad de Murcia, pronuncia la séptima ponencia sobre «La nueva política y la maternidad consciente» ${ }^{14}$. El tercer jurista es Quintiliano Saldaña, catedrático de Derecho en la Facultad de Derecho de Madrid. Favorable a la Liga Mundial para la Reforma Sexual creada en 1928 por Havelock Ellis, Bertrand Russel y George Bernard Shaw, su conferencia sobre «Eugenesia y Derecho» estaba prevista inicialmente para el curso 21 («Derecho y eugenesia») ${ }^{15}$.

El segundo grupo lo forman los médicos, entre los cuales figuran Roberto Novoa Santos, catedrático en la Facultad de Medicina de Madrid, quien pronuncia la primera ponencia, titulada «El sentido agresivo y canibalístico de la sexualidad» ${ }^{16}$; Gonzalo Lafora, miembro del Instituto Cajal, y autor de la sexta ponencia sobre «Pedagogía sexual, la educación sexual en la pubertad» ${ }^{17}$; César Juarros y Ortega, catedrático de psiquiatría, miembro de la Academia Nacional de Medicina, director de la «Escuela Central de Anormales», quien in-

13 Torrubiano, Jaime: «El Cristianismo es el mejor auxiliar de la Ciencia eugénica», Ibid., t. I, pp. 60-84.

14 RUIZ-FUNES, Mariano: «La nueva política y la maternidad consciente», Ibid., t. I, pp. 124-137.

15 SALDAÑA, Quintiliano: «Eugenesia y Derecho», Ibid., t. II, pp. 170-179.

16 Novoa SANTOS, R.: «El sentido agresivo y canibalístico de la Sexualidad», Ibid., t. I, pp. 18-31.

17 LAforA, Gonzalo R.: «Pedagogía Sexual», Ibid., t. I, pp. 107-123. 
terviene detenidamente en un curso técnico sobre lo que es su preocupación permanente, «El amor venal» ${ }^{18}$. Su contribución estaba prevista inicialmente para el curso $n^{\circ} 8$ sobre abolicionismo. Recordemos que Juarros fue diputado en Cortes en 1931, y que llamó allí la atención con sus propuestas revolucionarias acerca de la educación sexual y el aborto. Intervienen también José María Otaola, ginecólogo, director de la especialidad en la Gaceta Médica Española, quien debía hablar en el curso relativo a "Ginecología y obstétrica" sobre "Concepción y anticoncepción» ${ }^{19}$, y Francisco Haro, ginecólogo, de la Beneficencia municipal de Madrid, quien tenía prevista una contribución en el mismo apartado que Otaola y sobre el mismo tema: «Concepción y anticoncepción» ${ }^{20}$.

Entre los publicistas y escritores, entonces más o menos conocidos, figuran Matilde de la Torre, cuya ponencia sobre "Feminismo y Pacifismo» figura en segunda posición ${ }^{21}$, Pío Baroja, quien se encargaba de la cuarta ponencia en torno «al tema sexual en la Literatura» ${ }^{22}$, Ramón J. Sender, quien intervino después de Baroja, en la quinta ponencia titulada «Reflexiones sobre el Amor»23, Gonzalo de Reparaz, cuya contribución acerca de «El Amor en Oriente» tenía que darse en el curso sobre "Historia y eugenesia» ${ }^{24}$, y por fin, la joven Juana Capdevielle, doctora en Filosofía y Letras, quien se expresó sobre «El problema del Amor en el ambiente universitario» ${ }^{25}$. Su contribución estaba inicialmente prevista para el curso 20 relativo a «Pedagogía y eugenesia».

A pesar de las divergencias y de la variedad de su formación, de sus preocupaciones, de sus edades y de su sexo (hay dos mujeres en esta muestra, y hay tres en el corpus completo con Hildegart que intervino acerca del birth control y que decidí apartar por ser muy técnica su contribución), los trece participantes contribuyen todos en mayor o menor medida en la reflexión sobre la identidad femenina y su «devenir»a través de las cuestiones de la relación sexual y de la maternidad.

Se puede observar en las distintas intervenciones tres tipos de preocupaciones que además pueden convivir en el mismo conferenciante ya que participan de niveles distintos de preocupaciones y que el conferenciante que las formulas no siempre percibe la contradicción: la búsqueda, en el protocolo supuestamente natural e instintivo que rige el acto sexual, de los elementos básicos de la diferenciación sexual que, al definir una identidad femenina sometida, garantizaría la perennidad de la identidad masculina; la afirmación de una iden-

18 JUARros, César: «El amor venal», Ibid., t. I, pp. 245-272.

19 OtAOLA, J. M.: «Concepción y anticoncepción», Ibid., t. I, pp. 273-309.

20 HARO, F.: «Concepción y anticoncepción», Ibid., t. I, pp. 310-366.

21 De LA TORRE, Matilde: «Feminismo y Pacifismo», Ibid., t. I, pp. 33-59.

22 BAROJA, Pío: «El tema sexual en la Literatura», Ibid., t. I, pp. 85-92.

23 SENDER, Ramón J.: «Reflexiones sobre el Amor», Ibid., t. I, pp. 93-106.

24 DE RePARAZ, Gonzalo: «El amor en Oriente», Ibid., t. II, pp. 228-256.

25 CAPDevielle, Juana: «El problema del amor en el ambiente universitario», lbid., t. II, pp. 274-292.

Hispania, LXIV/3, núm. 218 (2004) 1003-1026 
tidad femenina basada en la capacidad procreadora que determinaría unos rasgos del carácter, sin hacer de la maternidad la única forma de realización de dicha identidad; la voluntad deliberada y a veces patética de proclamar la igualdad de los sexos sin abandonar el culto ancestral de la dominación masculina, y la dificultad para conciliar las igualdades jurídica, cívica, intelectual, etc. con la diferencia fisiológica y amorosa.

\section{DIFERENCIACIÓN SEXUAL E IDENTIDAD}

Con su conferencia provocadora sobre las componente caníbales del atractivo sexual, el médico Novoa Santos rompe inmediatamente con la mojigatería impuesta cinco años antes por el conservadurismo de la dictadura, y se dedica jocosamente a lo que algunos calificarían sin vacilar de "regodeo pornográfico». Según él, el acto amoroso equivale simbólicamente a la ingerencia y digestión del ser deseado (en este caso, la mujer, necesariamente vencida en esta lucha por la vida). Según esta tesis, la mujer dirigiría su golosa agresividad hacia el niño. La demostración que se conforma, en un primer momento, con confirmar la tesis tranquilizadora de la dominación histórica de las mujeres por los hombres, revela a la vuelta de una frase su segundo objetivo, más angustioso para la identidad masculina: imponer la idea de que en un mismo individuo (naturalmente bisexual) la componente masculina, siempre más fuerte y dominadora, tiende a sustituir a la componente femenina, incluso en las mujeres.

Para Roberto Novoa Santos, la humanidad ha alcanzado un clímax:

«Hasta el momento presente, todo cuanto nuestra mirada alcanza - mirando al dorso, a la espalda - nos viene a demostrar que esta evolución sexual de la Humanidad se ha realizado en el sentido de una mayor diferenciación sexual. La mujer se ha tornado más mujer, más cada día, y el hombre más varón cada día también [...] pero en esta dimensión del tiempo, en este futuro próximo o remoto, ¿qué nos aguarda, qué es, señores, lo que va a acaecer?»»26.

En su opinión, la humanidad ya no puede seguir en la vía de una diferenciación sexual cada vez más grande. Tampoco puede (esto es lo que le horroriza) ir hacia la conjunta virilización de los hombres y de las mujeres. Porque en su opinión, las manifestaciones de emancipación son muestras de virilización que ponen en tela de juicio la identidad masculina y la diferencia de los sexos:

«La mujer es cada vez más fuerte y de más talento, la mujer genial, enciclopédica, es precisamente por aquello que la mujer tiene siempre de hombrecito [...] de tal manera que la mujer se supera siempre, porque es en ella el transformador, el macho, que impera en ella como elemento agresivo y combatible» ${ }^{27}$.

\footnotetext{
26 Novoa Santos, R.: «El sentido agresivo y canibalístico de la Sexualidad», op. cit., p. 28.

27 Ibid., p. 29.
} 
Ante la angustia que produce el espectáculo ineludible de la emancipación que él llama «virilización», el profesor Novoa Santos se niega a desarrollar un discurso antifeminista y reaccionario, y resuelve la angustia de la pérdida de la identidad con la ayuda de una teoría de la vuelta a los orígenes andróginos por la que aboga y que no es sino la sublimación de la teoría de la complementariedad que oculta el rechazo de la pérdida de la hegemonía masculina.

Ramón J. Sender es presa de la misma angustia y del mismo dilema de hombre de izquierda dividido entre su virilidad inquieta y su voluntad feminista. Igualmente provocador, se hace el defensor incondicional del instinto en cuestiones de relaciones amorosas. Esto le permite, siguiendo la vía de Novoa Santos, hacer hincapié en la diferencia natural y fundamental de los sexos, diferencia que debería condicionar las relaciones estrictamente sexuales como también las relaciones sociales y amorosas:

«El instinto tiene mucho más desarrollados los recursos auxiliares en el hombre que en la mujer. El hombre, por naturaleza, debe conquistar a la mujer y poseerla. Para la conquista, necesita desarrollar todo género de medios de facilitación. Busca la mujer como un fin. El instinto no es probablemente el mismo en el hombre que en la mujer. Puede que la diferencia se pudiera señalar llamando incorrectamente al instinto masculino erótico, y al femenino, genésico. El hombre busca a la mujer. La mujer busca en cambio, el hijo [...]» ${ }^{28}$.

Se encuentra de nuevo aquí, y no es nada sorprendente, la idea de la dominación masculina y el tópico de una diferencia fundamental de naturaleza entre el instinto sexual masculino y el instinto sexual femenino. En esto, Sender se distingue de Novoa Santos que ve el mismo instinto (caníbal) en la relación hombre-mujer y en la relación madre-hijo. Sender cree en cambio en una diferencia más elemental todavía, que opondría de manera definitiva y radical feminidad y erotismo, y excluiría de éste la identidad femenina. Sin embargo, Sender reconoce implícitamente que a veces la pulsión erótica puede entrar en conflicto con la pulsión materna que es, en todo caso, la base de la identidad femenina:

«Las condiciones económicas y morales que padecemos hacen que muchas mujeres perfectamente dotadas por la Naturaleza y con el instinto en acecho y en ansiedad, no se entreguen al hombre que han elegido, y acepten, en cambio, antes que perder la posibilidad de ser madres y de realizar su misión, a otro cualquiera» 29 .

Según Sender, maternidad y feminidad mantienen relaciones estrechas:

28 SENDER, Ramón J.: «Reflexiones sobre el Amor», op. cit., p. 101.

29 Ibid., p. 102.

Hispania, LXIV/3, núm. 218 (2004) 1003-1026 
«Algunos fenómenos que tienen por causa la desviación del instinto - la desviación no lleva a lo patológico como la represión total- se pueden ofrecer por ejemplo en el caso de la esposa que no es madre y que al tropezar con esa anomalía elimina poco a poco de sus preocupaciones al marido. En cambio, la mujer cuyo instinto genésico se cumple con la fecundación admira al padre durante el embarazo, le atribuye el éxito del parto feliz y le sigue admirando después en la belleza o en el talento del hijo»30.

Sin embargo, Sender no cree en una diferencia de los caracteres basada en la diferencia sexual:

«Sobre las inhibiciones naturales del instinto va plasmado el carácter sexual del individuo, que es un factor importante, aunque yo no creo que sea decisivo en la formación de la personalidad, como han comprobado investigadores muy agudos en estos últimos años - Yung y Adler-. En la perfección viril o femenina, ya el carácter se desenvuelve sin nuevas inhibiciones ni alienaciones»3.1.

Esto le lleva a considerar que la pareja ideal tiene su fundamento en un atractivo físico recíproco al que se añade una humana simpatía independiente del sexo:

«Reciprocidad de deseo en el instinto, de simpatía en el carácter. Esta simpatía produce, con la convivencia, la ternura [...]. La reciprocidad del deseo y la posesión normal refuerzan esa ternura, cuya aparición en el amor da a éste su plenitud — viril en el hombre y femenina en la mujer- por una especie de gratitud fisiológica que se profesan los instintos en pleno cumplimiento de su misión»32.

Por su parte, César Juarros, que se interesa por la sexualidad masculina y la prostitución, evoca también como otros la sexualidad femenina en términos de oposición actividad-pasividad. Según él, en el orden natural, la feminidad debe situarse del lado de la pasividad, y la virilidad del lado de la actividad. Pero, ¿cómo interpretar, en la práctica prostitucional, la iniciativa femenina?:

«Ninguno de vosotros ignora que ha querido la naturaleza que el hombre sea activo en sus manifestaciones sexuales y la mujer pasiva. El hábito del burdel invierte los términos, acostumbrando al hombre a que la mujer sea protagonista, esté al timón y conduzca la nave de las caricias, haciendo que el macho adquiera hábitos de pasividad, o lo que es lo mismo se femenice, y cuando encuentra una mujer casta, honesta, una virgen romántica e ilusionada, rindiendo culto a aquellas condiciones, se muestra pasivo el que ya no puede ser activo, porque se habi-

\footnotetext{
30 Ibid.

31 Ibid., p. 105.

32 Ibid.
} 
tuó a la ramera pasividad, y calificando a la virgen de sosa y fría, vuelve a recordar a la mujer del burdel»33.

La identidad masculina también pasa por estas definiciones:

"Yo tengo la firme convicción de que no hay mujer sosa sino en contadas excepciones de procedencia patológica. A la mujer sosa la ocurre como a la guitarra, que dicen que es mala cuando el que la tañe no sabe tocar. Muchas mujeres que sus amantes o sus maridos han considerado sosas, en manos hábiles- y sobre todo leales para el instinto- se han acreditado como todo lo contrario» 34 .

Como se puede comprobar, aquellos hombres buscan, a través de una definición de la identidad femenina, la confirmación de la realidad de una identidad masculina. Les cuesta trabajo admitir que la sexualidad femenina pueda disociarse de la maternidad. En su favor, podemos observar un real esfuerzo con vistas a encontrar un lugar para el reconocimiento de una identidad femenina digna y respetable, ni reducida a su estricta función reproductora, como lo hace el jurista Saldaña que aboga por un total intervencionismo para la procreación y niega a las mujeres el derecho a elegir su maternidad ${ }^{35}$, ni exaltada o sublimada como a veces se ha dado el caso a lo largo de la historia, así como lo recuerda Gonzalo de Reparaz:

«La historia del mundo está llena de Aspasias. Y no digamos la de España. Y la causa: la inferioridad legal de la mujer; el encierro; el equiparar el cuerpo femenino a una joya preciosa, por preciosa más buscada, por guardada más apetitosa, por apetitosa más dominadora de quién la busca.

Por eso el poder de una favorita en un harén pudo ser, y fue muchas veces, mayor que el de la esposa única, libre y asociada al hombre, ya que no de igual a igual, lo que según vamos viendo, sólo empieza a suceder en nuestros días con alguna relativa libertad»36;

«Así en las tierras orientales, la mujer ha dirigido el destino de las naciones, sea como amada por bella, sea como temida y respetada por madre. El amor sexual o el amor maternal mandaban»37.

Finalmente, el obstétrico Otaola es quien se interesa con más serenidad en los mecanismos sexuales femeninos, que estudia con estricto rigor clínico destacado de cualquier consideración afectiva o sentimental. Tal procedimiento, guiado exclusivamente por la fría descripción fisiológica, le permite rechazar ideas tópicas basadas, en su mayoría, en un poco fiable paralelo entre sexo femenino y sexo

\footnotetext{
33 JUARRos, César: «El amor venal», op. cit., p. 258.

34 Ibid.

35 SALDAN̄A, Quintiliano: «Eugenesia y Derecho», op. cit., p. 175.

36 De RePARAZ, Gonzalo: «El amor en Oriente», op. cit., p. 232.

37 Ibid., p. 250.
} 
masculino (orgasmo y fecundación, pene y clítoris). En su conferencia, aparecen pocas consideraciones que permitan, a partir de la realización del acto sexual, extrapolar alguna «identidad femenina» determinada por el sexo, salvo cuando revela, con simpatía además, toda la rebelión profundamente humana e identitaria que puede ocultarse detrás de ciertas formas de frigidez:

«Las enfermas sienten dentro de sí el «no quiero», el «no debo», y lo transforman por un «no puedo». El descubrir este interno «no» corresponde al psicoanálisis. En el tratamiento de estos trastornos, este «no puedo» también quiere decir «yo no quiero ser mujer» o «yo no quiero ser mujer para ti»; La frigidez nos muestra la progresiva disociación del hombre civilizado, su discordia en un quiero y no quiero, su interno desgarramiento, su juego ante si mismo y su lucha consigo mismo» 38 .

\section{«MATERNIDAD CONSCIENTE», BIRTH CONTROL Y SEXUALIDAD}

Desde luego, ninguno de los congresistas pone en tela de juicio -lo que se comprende en un congreso sobre eugenismo-, la importancia de la procreación en las relaciones que rigen a hombres y mujeres. Ninguno pone en tela de juicio la maternidad como base de la identidad femenina. Algunos van incluso hasta afirmar que si la identidad femenina se basa en su capacidad procreadora, y por esta misma capacidad es por lo que se impone como una necesidad el derecho a controlar los nacimientos.

Así es cómo el jurista Mariano Ruiz Funes muestra claramente que la exigencia de maternidades numerosas daña gravemente la salud de las mujeres:

«Decir que la Constitución protege la maternidad porque con la maternidad protege aquellas proles numerosas y aquellas madres que las producen, perdiendo en la empresa incluso su condición de mujer, es afirmar que la Constitución no es la superlegalidad de un principio jurídico humano; es sostener que la Constitución es la protección de un abuso contra la propia maternidad y contra el propio organismo de la mujer $\gg 39$.

O sea que, al exigir numerosas maternidades, la sociedad va en contra de la feminidad misma:

«[La maternidad] es temida no en cuanto al hecho mismo de la maternidad, sí en cuanto a la multiplicación de esta maternidad, que puede suprimir, puede llegar a suprimir, puede llegar a hacer ineficaces todas las esencias íntimas del alma de la mujer, que de otro modo daría su adecuado rendimiento sentimental, espiritual y social.

38 OTAOL.A, J. M.: «Concepción y anticoncepción», op. cit., p. 281.

39 RUIZ-FUNES, Mariano: «La nueva política y la maternidad consciente», op. cit., p. 129. 
Cuando se dice que el hombre que se casa entrega rehenes a la suerte, se contempla otra parte del problema, se tiene una visión parcial del tema. Efectivamente, por toda pareja que se une, se entregan rehenes a la suerte; pero hay que preguntarse, llegado este momento, quién entrega de la pareja humana más rehenes a la suerte; si el hombre o la mujer; la mujer que compromete muchas veces su vida en el dramático sacrificio de la maternidad y que puede, por la multiplicación de este sacrificio, llegar a perder su propia condición de mujer» ${ }^{40}$.

Ruiz Funes es uno de los pocos participantes capaces de señalar así con tanta crudeza los efectos agresivos y dañinos de la maternidad.

Más abstracta por sus demostraciones que por sus convicciones, Matilde de la Torre desarrolla por su parte una concepción historicista y evolucionista de la cuestión. En su opinión, la moral social que tiende a exigir un gran número de niños fue válida y eficaz en tiempos remotos, pero ahora ya resulta anacrónica ${ }^{41}$. De la misma manera que el nacimiento del sentimiento amoroso respondería a una necesidad coyuntural de la especie que, en algún momento, necesitaría proceder a alguna forma de selección ${ }^{42}$, la maternidad prolífica también resultaría de una necesidad de la especie en un primer tiempo, de las naciones en un segundo momento, y de una necesidad industrial, para terminar.

Matilde de la Torre plantea entonces la pregunta: «¿Hasta dónde es lícito procrear en las condiciones actuales de la sociedad?» ${ }^{43}$. Y añade que «las mujeres actuales serán las primeras en comprender el sentimiento del Nuevo Derecho que defendemos para ellas». Dicho de otro modo, es porque son madres potenciales por lo que las mujeres siempre han tenido el sentido de las necesidades de la especie, según los tiempos y según las circunstancias. De ahí su relación fuerte 'con el pacifismo. Matilde de la Torre rechaza por lo tanto un pacifismo pretendidamente femenino e inmutablemente natural:

«Las mujeres no somos pacifistas por doctrina y ni aun por sentimiento. Es más: las hembras en general no son pacifistas: son hembras nada más»44.

Feminista e intelectual, la publicista reivindica una identidad femenina basada en la condición de hembra de la especie que posee naturalmente el instinto de lo que en un momento dado necesita dicha especie. Esta intuición es lo que realmente es positivo y lleva a las mujeres a militar hoy a favor de la «maternidad consciente" y del birth control.

Francisco de Haro reivindica igualmente para las mujeres el derecho a no procrear; incluso reivindica el deber, en ciertos casos, de no procrear ${ }^{45}$. Pero no

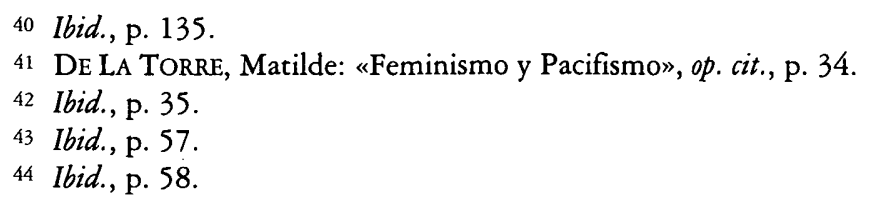


lo hace, como Matilde de la Torre, en nombre de una identidad femenina trascendente, sino en nombre de un derecho humano al gozo. Como el profesor Otaola, hace hincapié en la necesidad de disociar el orgasmo y la fecundación, y saca de ello la siguiente conclusión:

«La mujer, pues, tiene derecho a realizar su función sexual como el hombre, combinando, simultaneando el deleite, el placer, con la función procreadora, pero también tienen derecho a separar ambos elementos y rehuir, en momentos en que sólo al deleite quieran rendir tributo, todo lo relacionado con la reproducción, y si contra todo deseo de la mujer, contra todo despego por la maternidad ésta irrumpe contra viento y marea, ¿por qué no ha la mujer de tener derecho a intervenir con un aborto que le permita conservar su derecho a no ser madre?»46.

Las palabras de Francisco de Haro nos conducen a la tercera y última preocupación, la que consiste en proclamar la emancipación femenina y la diferencia en términos de identidad femenina.

\section{EMANCIPACIÓN FEMENINA Y EMANCIPACIÓN SEXUAL}

Condenando los falsos pudores que él considera como una trampa para engañar a las mujeres, Haro invita a estas últimas a tomar una parte activa en el combate eugénico:

«Y es verdad, la mujer, y buena prueba está dando en estas jornadas, tiene que tomar parte capital en estas enseñanzas, en estas campañas, rebelándose contra los que juegan con su pudor para utilizarle unas veces como escudo que impida llegar hasta ellas todas las medidas beneficiosas, y otras como venda que oculte a su vista las concupiscencias varoniles que ella después va a sufrir» ${ }^{47}$.

El jesuita Torrubiano Ripoll le enseñó la vía en su ponencia. El anticonformismo y la independencia que revelan las palabras de aquel sacerdote obligan a la simpatía y al respeto. Se niega a aferrarse en el tema del matrimonio religioso. No quiere considerar que sólo la total abstinencia merece el nombre de castidad. Desea para los hombres como para las mujeres una misma castidad y una misma «virginidad». Cuando condena el neomaltusianismo, es porque ve en esta práctica un comportamiento egoísta que no se da, en cambio, afirma Torrubiano Ripol, en el birth control que él defiende. Porque opina que estas dos prácticas tienen significados éticos totalmente opuestos:

45 HARO, F.: «Concepción y anticoncepción», op. cit., p. 335.

46 Ibid., p. 364.

47 Ibid., p. 331. 
«La norma de los neomaltusianistas absolutos es el placer a todo trance, sin deberes y sin riesgos, sin responsabilidades y sin cargas, sin preocupación siquiera por la posibilidad de un nuevo ser que pueda restarles parte de su bienestar material $[. .$.$] con esos seres refinadamente egoístas, enemigos de su salud y de su con-$ ciencia, de la vida y de la patria, tendrá que ver el ginecólogo, el neurólogo, el médico alienista, el moralista, el sociólogo, y tal vez el poder público; pero de ningún modo el eugenista.

Con los profesionales de la virginidad y del celibato por motivos de orden superior podrá discutirse si es lícita o no es lícita su postura y su abstinencia [...] pero tampoco tiene nada que ver con ellos el eugenista»48;

«Sólo los sufrimientos normalmente intolerables que hayan de sobrevenir en el progenitor o en la familia como consecuencia de una nueva prole, o la seguridad moral de una descendencia totalmente inútil o gravemente desgraciada, pueden legitimar, en cada caso concreto debidamente estudiado, la limitación de la prole por alguno de los medios anticoncepcionales conocidos, en cada caso debidamente-seleccionados, y aun tal vez, con grandísimas restricciones, -lo digo con recelo y con zozobra- por el aborto» 49 .

En cuanto a Pío Baroja, que recuerda insistentemente que el amor es un asunto complicado, que no puede reducirse a una mera cuestión fisiológica, denuncia jocosamente los egoísmos masculinos, mostrándose tranquilamente feminista e indignado de la condición impuesta a las mujeres por la sociedad:

«Esto, naturalmente, es muy cómodo para el hombre; pero yo no sé si será fácil encontrar este mirlo blanco. Desde el punto de vista de la mujer, no me parece un ideal muy halagüeño ese de ser siempre el paño de lágrimas del hombre que en algunas ocasiones, raras, podrá ser un tipo noble y extraordinario, pero que en la mayoría de los casos puede ser un personaje mezquino, cominero y ridículo.

Está muy bien para el hombre encontrar una mujer fuerte, sana e inteligente, y para la mujer un hombre noble y trabajador; pero éstos son específicos que no se encuentran en las boticas» 50 .

\section{Insiste Baroja en el peso de la Historia:}

«En mi tiempo la vida de la juventud era bastante mediocre. Las mujeres decentes y distinguidas eran como plazas fuertes: atrincheradas y amuralladas. Llevaban un corsé que era como la muralla de la China o el baluarte de Verdun. Si por casualidad uno ponía la mano en su talle, encontraba una coraza tan dura como la que podía llevar a las Cruzadas Godofredo de Bouillón.

\footnotetext{
48 TORRUBIANO, Jaime: «El Cristianismo es el mejor auxiliar de la Ciencia eugénica», op. cit.,

49 Ibid., p. 71

50 BAROJA, Pío: «El tema sexual en la Literatura», op. cit., p. 88.
} p. 69. 
Si uno pretendía entrar en relación con uno de aquellos Verdunes vivos, le contestaban varios días o semanas Si y No, como Cristo nos enseña.

Únicamente si podía uno presentar en el estandarte un sueldecito o una renta, bajaba el puente levadizo del castillo y se parlamentaba.

Lo que produce la dificultad del problema sexual no es la fisiología ni la patología humana, sino su carácter mixto, complejo, formado por la Historia durante miles de años $[\ldots] \gg 51$.

Por fin, Baroja ridiculiza el antiguo y lamentable debate sobre la tendencia natural masculina a la monogamia o poligamia:

«Se pone a un hombre normal con una profesión que le guste y le obligue a trabajar mucho, una mujer amable y familiar, y será monógamo. Se le deja rico, desocupado, en un ambiente laxo, con una mujer poco agradable, y será polígamo. El hombre es ya demasiado viejo y demasiado elástico para ser fundamental y exclusivamente una cosa u otra.

Lo mismo se puede asegurar de la mujer que será monógama (si se puede llamar así a una mujer que vive sólo con un hombre) o poliándrica, según el ambiente en qué se desarrolle52.

La joven Juana Capdevielle tiene la última palabra en aquellas jornadas sobre eugenesia. Al señalar el poco camino recorrido por las instituciones y las mentalidades a lo largo de los años anteriores, Juana Capdevielle revela al público la madurez, la independencia intelectual, y la modernidad adquiridas en el mismo periodo por una reducida franja de la juventud universitaria femenina. Para empezar, Juana Capdevielle lleva tranquilamente la contraria a todos aquellos notables que han mostrado cierta compasión para con las mujeres y su condición. Orgullosamente, Juana Capdevielle reivindica su feminidad como una plena realización:

«Ni siquiera la espléndida disertación de hace unos días de vuestro maestro señor Novoa Santos ha podido hacerme comprender la inferioridad femenina, la desgracia de ser mujer, y creo que el destino de ésta en el Mundo es algo tan maravilloso que si yo hubiera de vivir otra vida y me preguntasen previamente mi opinión, pediría resueltamente volver a ser mujer»\$3.

Inmediatamente después, recuerda los mecanismos sociales de la doble ética tradicional que opone la santa esposa y los amores venales, y denuncia con mucho humor y gallardía la trampa en la que llevan tanto tiempo cogidas las mujeres casadas:

51 Ibid., pp. 88-89.

52 Ibid., p. 90.

s3 CAPDEVIElLE, Juana: «El problema del amor en el ambiente universitario», op. cit., p. 276. 
«Todos recordaréis el personaje de Benavente, cuya mujer se da, además, por muy satisfecha, el día en que, gastado, explotado y despreciado por las otras vuelva a ella -iqué remedio! - ya que no le queda nadie que le haga caso, y ella entonces exclama con voz declamatoria: «iMis rosas de otoño!»54.

También se indigna, con el mismo tono, del desprecio de la literatura «donjunanesca» universal hacia las jóvenes enamoradas (y cita al respecto a Kierkegaard).

El comportamiento de los hombres jóvenes de su generación se basa en aquellos preceptos morales que ella considera anticuadísimos:

«Hijos de quienes entendían de una manera tan pintoresca la moral, los jóvenes del día se han oído decir en todos los tonos, que hay que empezar a divertirse muy pronto para demostrar que se es muy hombre. Como, por otra parte, no conciben otra forma de diversión que la que les han inculcado esos absurdos padres, resulta, pena da decirlo, que gran parte de ellos cultivan, como sus antecesores, ese que llamaré — permitidme el eufemismo — amor de tarifa [... $\}$ »55.

Juana Capdevielle considera que este tipo de relaciones mantiene y perenniza la irresponsabilidad y la vanidad masculina:

«¿Quién se ha planteado [...] el problema de la parcela de responsabilidad que le corresponde en el gran crimen colectivo que se comete con la prostitución? [...] Frente a pobres mujeres embrutecidas; las mínimas cualidades de inteligencia, de finura, de sensibilidad adquieren proporciones apoteósicas»\$5.

Más original, según ella, sería el nuevo Don Juan, el que atrapa a sus víctimas con el desafío de la modernidad o del intelectualismo. Juana Capdevielle ridiculiza la ingenuidad de aquellos jóvenes conquistadores:

«Es de ver la cara absorta con que ante la negativa, no ya indignada, sino simplemente despectiva, de una mujer responsable, exclaman contristados «¿Pero cómo, si es usted una mujer moderna e intelectual, tiene el valor de rechazarme?». Y dan ganas de reírse y contestarles: «Por una razón aplastante: que es usted muy feo y muy poco interesante» 57 .

De esta manera, nuestra joven universitaria reivindica tranquilamente un saber-negarse-a-la-aventura, que nada tiene que ver con la moral social y que resulta exclusivamente de un derecho "natural», el derecho a elegir libremente a su pareja.

54 Ibid., p. 277.

ss Ibid., p. 278.

56 Ibid.

57 Ibid.

Hispania, LXIV/3, núm. 218 (2004) 1003-1026 
Son presas privilegiadas de aquellos jóvenes conquistadores las jóvenes que sustituyen nuevos prejuicios a los prejuicios antiguos y que, en uno y otro caso, van en contra de sus aspiraciones más profundas para atraerse el favor de los varones. Indignada por la hipocresía del flirteo, Juana Capdevielle afirma sus ideas y sus convicciones en términos de sexualidad, considerando que sólo una forma de frigidez hace posible el juego con los límites, y que el amor es compatible con la ética única y exclusivamente cuando existe una total sinceridad en la plenitud de su realización:

«Creo que todos entenderéis a quién me refiero: a esas que llaman en francés «demi-vierges», a esa profesional del flirt, cuya frialdad le permite bordear en todo momento la aventura definitiva, sabiendo en todo momento basta dónde puede ir... y esto no es tolerable, esto no es admisible: en amor se va o no se va; lo que no se puede hacer honradamente, limpiamente, es quedarse a la mitad del camino» 58 .

Finalmente, Juana Capdevielle milita a favor de una vida amorosa completa y sincera, en la que el abandono de las reglas sociales tradicionales no implica el desorden amoroso, sino al contrario la reconciliación de las aspiraciones del cuerpo y del espíritu, con el más riguroso respeto de la dignidad. Pero sus conclusiones son poco optimistas. La sociedad española de los primeros años de la Segunda República, según ella, no está dispuesta todavía a acoger sin miramientos a aquella mujer «consciente y responsable» que empezaba a construirse, y la suerte de Juana Capdevielle, cuyo cadáver fue encontrado tres años después destrozado y humillado en una cuneta en los primeros meses de la Guerra civil, muestra horriblemente hasta qué punto tenía razón la entonces joven, entusiasta, pero tan lúcida, doctora de filosofía y letras:

«Cuando entre tantas neurótica, hipócritas y cobardes surge una mujer sana, sincera y responsable, que a todo trance quiere mantener su libertad, pero que tiene conciencia de su dignidad, que busca afanosamente las alegrías que la vida puede darle, pero que no rehuye los deberes que le impone, su situación es poco menos que insostenible: nos la tachan de atrevida, otros de timorata; en la derecha la llaman cínica, y en la izquierda hipócrita; y si los que la halagan consiguen ganar su confianza, luego, mas tarde, la dejan para casarse con la burguesita tradicional, sin comprender que es de entre éstas que surgió madame Bovary, y que una mujer conciente y responsable es precisamente la que no engaña, porque podrá dejar de querer, pero no es capaz de mentir, pues la que siguió en su vida una trayectoria de limpieza podrá abandonar a su marido, pero no llega nunca a la odiosa promiscuidad del adulterio» 59 .

$\begin{array}{ll}58 & \text { Ibid., p. } 282 . \\ 59 & \text { Ibid., p. } 284 .\end{array}$

Hispania, LXIV/3, núm. 218 (2004) 1003-1026 
Juana Capdevielle lo reconoce al final de su contribución: es muy difícil, en esta sociedad cuyos esquemas se transmiten de generación en generación, echar abajo en unos cuantos años los prejuicios, los tópicos, las pautas de comportamiento, para recuperar el tiempo y poner las prácticas al compás de la modernidad.

Las dificultades y las incoherencias que apuntó la joven universitaria entre los hombres y mujeres tienen su reflejo en las palabras de los congresistas de aquellas Jornadas eugénicas. A estos hombres de izquierda, en su mayoría, les cuesta trabajo, sin embargo, aceptar que la liberalización de las costumbres en materia sexual no significa la pérdida irremediable de las identidades femenina y masculina, identidades sobre las cuales se basa el modelo único de las diferentes construcciones sociales entonces concebidas y concebibles.

Sin embargo, un real esfuerzo fue realizado a lo largo de las Jornadas para ir más allá de los prejuicios moralizadores y biologizantes, para proponer soluciones susceptibles de conciliar los intereses individuales y colectivos, para debatir con serenidad de los límites éticos del intervencionismo jurídico y médico. 